\title{
CALDERÓN'S INVERSE PROBLEM WITH A FINITE NUMBER OF MEASUREMENTS
}

\author{
GIOVANNI S. ALBERTI ${ }^{\circledR}$ and MATTEO SANTACESARIA ${ }^{\circledR}$ \\ Department of Mathematics, University of Genoa, Via Dodecaneso 35, 16146 Genova, Italy; \\ email: giovanni.alberti@unige.it, matteo.santacesaria@unige.it
}

Received 6 January 2019; accepted 11 September 2019

\begin{abstract}
We prove that an $L^{\infty}$ potential in the Schrödinger equation in three and higher dimensions can be uniquely determined from a finite number of boundary measurements, provided it belongs to a known finite dimensional subspace $\mathcal{W}$. As a corollary, we obtain a similar result for Calderón's inverse conductivity problem. Lipschitz stability estimates and a globally convergent nonlinear reconstruction algorithm for both inverse problems are also presented. These are the first results on global uniqueness, stability and reconstruction for nonlinear inverse boundary value problems with finitely many measurements. We also discuss a few relevant examples of finite dimensional subspaces $\mathcal{W}$, including bandlimited and piecewise constant potentials, and explicitly compute the number of required measurements as a function of $\operatorname{dim} \mathcal{W}$.
\end{abstract}

2010 Mathematics Subject Classification: 35R30 (primary); 42C40, 94A20 (secondary)

\section{Introduction and main results}

Consider the Schrödinger equation

$$
(-\Delta+q) u=0 \quad \text { in } \Omega,
$$

where $\Omega \subseteq \mathbb{R}^{d}, d \geqslant 3$, is an open bounded domain with Lipschitz boundary and $q \in L^{\infty}(\Omega)$ is a potential. Assuming that

$$
0 \text { is not a Dirichlet eigenvalue for }-\Delta+q \text { in } \Omega \text {, }
$$

it is possible to define the Dirichlet-to-Neumann (DN) map

$$
\Lambda_{q}:\left.\left.u\right|_{\partial \Omega} \mapsto \frac{\partial u}{\partial v}\right|_{\partial \Omega}
$$

(c) The Author(s) 2019. This is an Open Access article, distributed under the terms of the Creative Commons Attribution licence (http://creativecommons.org/licenses/by/4.0/), which permits unrestricted re-use, distribution, and reproduction in any medium, provided the original work is properly cited. 
where $v$ is the unit outward normal to $\partial \Omega$, defined as an operator $\Lambda_{q}: H^{1 / 2}(\partial \Omega) \rightarrow H^{-1 / 2}(\partial \Omega)$.

1.1. Global uniqueness. The Gel'fand-Calderón's inverse problem asks if it is possible to determine the potential $q$ from the knowledge of its associated DN map $\Lambda_{q}$. A more realistic version of the problem is the following: assuming that $q$ belongs to a known finite dimensional subspace of $L^{\infty}(\Omega)$, is it uniquely determined from a finite number of boundary measurements? Our main result gives a positive answer to this question.

THEOREM 1. Take $d \geqslant 3$ and let $\Omega \subseteq \mathbb{R}^{d}$ be a bounded Lipschitz domain and $\mathcal{W} \subseteq L^{\infty}(\Omega)$ be a finite dimensional subspace. There exists $N \in \mathbb{N}$ such that for any $R>0$ and $q_{1} \in \mathcal{W}$ satisfying $\left\|q_{1}\right\|_{L^{\infty}(\Omega)} \leqslant R$ and (2), the following is true.

There exist $\left\{f_{l}\right\}_{l=1}^{N} \subseteq H^{1 / 2}(\partial \Omega)$ such that for any $q_{2} \in \mathcal{W}$ satisfying $\left\|q_{2}\right\|_{L^{\infty}(\Omega)} \leqslant R$ and (2):

$$
\text { if } \Lambda_{q_{1}} f_{l}=\Lambda_{q_{2}} f_{l} \text { for } l=1, \ldots, N \text {, then } q_{1}=q_{2} \text {. }
$$

REMARK 1. The dependence of $N$ on $\mathcal{W}$ is explicit in the proof, see (14). Thus, in principle, it is always possible to determine $N$ given the subspace $\mathcal{W}$ (see Section 4 for three relevant examples).

REMARK 2. The assumption that 0 is not a Dirichlet eigenvalue for $-\Delta+q$ in $\Omega$ has been made only to define $\Lambda_{q}$, which simplifies the exposition, and it can be removed. See Remark 3 for more details.

Theorem 1 readily yields a similar result for Calderón's inverse conductivity problem [18], which concerns the determination of an electrical conductivity $\sigma \in$ $L^{\infty}(\Omega)$ satisfying

$$
\lambda^{-1} \leqslant \sigma \leqslant \lambda \quad \text { almost everywhere in } \Omega
$$

for some $\lambda>1$, from the DN map

$$
\Lambda_{\sigma}:\left.\left.u\right|_{\partial \Omega} \mapsto \sigma \frac{\partial u}{\partial v}\right|_{\partial \Omega},
$$

where $u$ solves the conductivity equation $-\operatorname{div}(\sigma \nabla u)=0$ in $\Omega$. This is the mathematical model for electrical impedance tomography (EIT).

COROLlary 1. Take $d \geqslant 3$ and let $\Omega \subseteq \mathbb{R}^{d}$ be a bounded Lipschitz domain and $\mathcal{W} \subseteq L^{\infty}(\Omega)$ be a finite dimensional subspace. There exists $N \in \mathbb{N}$ such that for 
any $\lambda>1$ and $\sigma_{1} \in W^{2, \infty}(\Omega)$ satisfying (3) and such that $\Delta \sqrt{\sigma_{1}} / \sqrt{\sigma_{1}} \in \mathcal{W}$ and $\sigma_{1}=1$ in a neighborhood of $\partial \Omega$, the following is true.

There exist $\left\{f_{l}\right\}_{l=1}^{N} \subseteq H^{1 / 2}(\partial \Omega)$ such that for any $\sigma_{2} \in W^{2, \infty}(\Omega)$ satisfying (3) and such that $\Delta \sqrt{\sigma_{2}} / \sqrt{\sigma_{2}} \in \mathcal{W}$ and $\sigma_{2}=1$ in a neighborhood of $\partial \Omega$ :

$$
\text { if } \Lambda_{\sigma_{1}} f_{l}=\Lambda_{\sigma_{2}} f_{l} \text { for } l=1, \ldots, N \text {, then } \sigma_{1}=\sigma_{2} \text {. }
$$

To our knowledge, these are the first uniqueness results for the Gel'fandCalderón and Calderón problems with a finite number of measurements. The only previous result of this kind is [24], where it was shown that a single boundary measurement was enough to determine a piecewise constant conductivity with discontinuities on a single convex polygon. All other uniqueness results rely on an infinite number of measurements, even for potentials belonging to known finite dimensional subspaces. Some fundamental contributions to the two problems include $[10,17,19,26,31,32,36,37,44]$ for global uniqueness and reconstruction, and $[6,15,20,39]$ for global stability. An interesting uniqueness result from a finite number of measurements is [16], for a related inverse problem.

1.2. Lipschitz stability and reconstruction. We are also able to prove Lipschitz stability estimates for the two problems.

THEOREM 2. Take $d \geqslant 3$ and let $\Omega \subseteq \mathbb{R}^{d}$ be a bounded Lipschitz domain and $\mathcal{W} \subseteq L^{\infty}(\Omega)$ be a finite dimensional subspace. There exists $N \in \mathbb{N}$ such that for every $R, \alpha>0$ and $q_{1} \in \mathcal{W}$ satisfying $\left\|q_{1}\right\|_{L^{\infty}(\Omega)} \leqslant R$ and (2), the following is true.

There exist $\left\{f_{l}\right\}_{l=1}^{N} \subseteq H^{1 / 2}(\partial \Omega)$ such that for every $q_{2} \in \mathcal{W}$ satisfying $\left\|q_{2}\right\|_{L^{\infty}(\Omega)} \leqslant R$ and (2), we have

$$
\left\|q_{2}-q_{1}\right\|_{L^{2}(\Omega)} \leqslant e^{C N^{1 / 2+\alpha}}\left\|\left(\Lambda_{q_{2}} f_{l}-\Lambda_{q_{1}} f_{l}\right)_{l=1}^{N}\right\|_{H^{-1 / 2}(\partial \Omega)^{N}}
$$

for some $C>0$ depending only on $\Omega, R$ and $\alpha$.

COROLlaRY 2. Take $d \geqslant 3$ and let $\Omega \subseteq \mathbb{R}^{d}$ be a bounded Lipschitz domain and $\mathcal{W} \subseteq L^{\infty}(\Omega)$ be a finite dimensional subspace. There exists $N \in \mathbb{N}$ such that for any $\lambda>1, \alpha>0$ and $\sigma_{1} \in W^{2, \infty}(\Omega)$ satisfying (3) and such that $\Delta \sqrt{\sigma_{1}} / \sqrt{\sigma_{1}} \in$ $\mathcal{W}$ and $\sigma_{1}=1$ in a neighborhood of $\partial \Omega$, the following is true.

There exist $\left\{f_{l}\right\}_{l=1}^{N} \subseteq H^{1 / 2}(\partial \Omega)$ such that for any $\sigma_{2} \in W^{2, \infty}(\Omega)$ satisfying (3) and such that $\Delta \sqrt{\sigma_{2}} / \sqrt{\sigma_{2}} \in \mathcal{W}$ and $\sigma_{2}=1$ in a neighborhood of $\partial \Omega$, we have

$$
\left\|\sigma_{2}-\sigma_{1}\right\|_{L^{2}(\Omega)} \leqslant e^{C N^{1 / 2+\alpha}}\left\|\left(\Lambda_{\sigma_{2}} f_{l}-\Lambda_{\sigma_{1}} f_{l}\right)_{l=1}^{N}\right\|_{H^{-1 / 2}(\partial \Omega)^{N}}
$$

for some $C>0$ depending only on $\Omega, \lambda$ and $\alpha$. 
These are the first stability estimates for the Gel'fand-Calderón and Calderón problems with a finite number of measurements. Lipschitz stability results have been previously known only when an infinite number of measurements are available [7-9, 11-14, 25]. The exponentially growing constant is coherent with the exponential instability of the problem $[21,28,33,42]$.

We finally employ the same ideas to present a new nonlinear iterative reconstruction algorithm for the two inverse problems and prove that it is globally convergent in Theorem 4 . Given $\left(f_{l}, \Lambda_{q} f_{l}\right)_{l=1}^{N}$, the algorithm constructs a sequence $q_{n} \rightarrow q$ in $L^{2}(\Omega)$ where $q_{0} \in \mathcal{W}$ is any initial guess. The algorithm converges exponentially and its stability is given by Theorem 2 and Corollary 2 . The details are presented in Section 3.

Note that this represents the first globally convergent iterative algorithm for the Gel'fand-Calderón and Calderón problems from a finite number of measurements. All reconstruction algorithms used so far have been either based on the full DN map, either locally convergent or with no proof of convergence. We refer to [34] for an extensive review on reconstruction methods for nonlinear inverse problems.

The strategy of the proof of Theorems 1 and 2 is as follows. We use Alessandrini's identity to transform the boundary data into integral measurements, and use complex geometrical optics (CGO) solutions to construct a nonlinear operator $U$, expressing the DN map in a more convenient form. We then write $U$ as $U=F+B$ where $F$ is the Fourier transform and $B$ is a nonlinear term. We show that $B$ is a contraction, provided the CGO solutions are constructed for sufficiently high complex frequencies. Finally, the problem is reduced to a fixedpoint problem involving a nonlinear Fourier transform.

1.3. Open questions and comments. This paper has been greatly motivated by potential applications of ideas coming from applied harmonic analysis and sampling theory to inverse problems in partial differential equations (PDE). This approach paves the way for several interesting research directions and open problems. Let us mention some of them.

- The inspiration for this paper came from a previous paper of the authors [4], in which the theory of compressed sensing (CS) was generalized to the infinite dimensional setting for linear operators which are not necessarily isometries, in order to make it more flexible for the applications to inverse problems in PDE. The current paper shows that the effects of the nonlinearity of the inverse problem may be mitigated by carefully selecting the measurements. Thus, we expect that the theory of CS may be applied to this nonlinear problem as well: this would give that the number of measurements needed is (substantially) proportional to the sparsity of the unknown. 
- In this paper, the boundary Dirichlet data $\left\{f_{l}\right\}_{l}$ are chosen dependently of the unknown potential $q$. It is natural to wonder whether, using the assumption that $q$ belongs to a finite dimensional space, it is possible to determine (a possibly larger number of) $\left\{f_{l}\right\}_{l}$ independently of $q$. A partial answer to this issue is given in the recent preprint [5].

- In Corollaries 1 and 2 we assumed that the conductivities are equal to 1 in a neighborhood of the boundary. In order to overcome this limitation, one would need to recover the boundary values of a conductivity and of its normal derivative from the boundary data. This is well understood when the full DN map is available $[35,36]$, but it is still open in case of a finite number of measurements.

- The results of this paper cannot be directly extended to the two dimensional case. In that setting, the reconstruction methods for the Gel'fand-Calderón and the Calderón problem are different from the one presented here, and it would be very interesting to study the problem with a finite number of measurements.

- In Section 4 we compute the number of required measurements $N$ for some choices of subspaces $\mathcal{W}$ : it would be interesting to study the optimality of these bounds and to derive similar estimates in other relevant cases.

- In this paper we consider the continuum model for these inverse boundary value problems. Possible extensions to more realistic and physical models (such as the complete electrode model for EIT) and the numerical analysis and implementation of the reconstruction algorithm presented may be investigated. This was studied in [27], after the appearance of the preprint of this work.

- It is natural to wonder whether $\mathcal{W}$ could be required to be only a finite dimensional submanifold of $L^{\infty}(\Omega)$ and not necessarily a linear subspace. As we point out in Remark 4, the current proof does not work in such generality, and new ideas are required.

- Finally, it is expected that the approach presented in this paper can be extended to other infinite dimensional inverse problems in PDE with finitely many measurements.

1.4. Structure of the paper. Section 2 contains the proofs of the results stated above. Section 3 presents the reconstruction algorithm and its convergence properties. In Section 4 we discuss some examples of subspaces $\mathcal{W}$, for which we compute explicitly the number of required measurements $N$ as a function of $\operatorname{dim} \mathcal{W}$. 


\section{Proofs}

Take $q \in L^{\infty}(\Omega)$ such that $\|q\|_{L^{\infty}(\Omega)} \leqslant R$. Given two boundary voltages $f$, $g \in H^{1 / 2}(\partial \Omega)$ we have Alessandrini's identity [6]:

$$
\left\langle g,\left(\Lambda_{q}-\Lambda_{0}\right) f\right\rangle_{H^{1 / 2}(\partial \Omega) \times H^{-1 / 2}(\partial \Omega)}=\int_{\Omega} q u_{g}^{0} u_{f} d x,
$$

where $u_{f}$ (respectively $u_{g}^{0}$ ) solves the Schrödinger equation (1) with potential $q$ (respectively 0 ) and Dirichlet data $f$ (respectively $g$ ). The quantity on the left of this identity is known since $\Lambda_{q} f$ is the boundary measurement corresponding to the chosen potential $f$ and $\Lambda_{0}$ is the DN map corresponding to the unperturbed Laplacian. This identity allows to transform the boundary data into measurements of scalar products between the unknown $q$ and some test functions to be suitably chosen.

REMARK 3. As anticipated in Remark 2, assumption (2) was made only to define $\Lambda_{q}$, but can be removed. Indeed, it is possible to consider a finite number of Cauchy data as boundary measurements, that is $\left(\left.u_{l}\right|_{\partial \Omega}, \partial u_{l} /\left.\partial v\right|_{\partial \Omega}\right)_{l=1}^{N}$, where $(-\Delta+q) u_{l}=0$ in $\Omega, l=1, \ldots, N$. In this case the same uniqueness and stability results hold, provided one makes use of the results of [29], where Alessandrini's type identities were obtained for a general impedance boundary map, also known as Robin-to-Robin map. This is a generalization of the DN map, and it can be defined even when 0 is a Dirichlet eigenvalue for the Schödinger operator.

Without loss of generality, we can assume that $\Omega \subseteq \mathbb{T}^{d}$, where $\mathbb{T}=[0,1]$, and in the following we will extend any function of $L^{\infty}(\Omega)$ to $L^{\infty}\left(\mathbb{T}^{d}\right)$ by zero. Fix an arbitrary parameter $p \in(d,+\infty)$. In the rest of this subsection, with an abuse of notation, several different positive constants depending only on $d, p$ and $R$ will be denoted by the same letter $c$.

We now construct a special class of solutions to (1) as in the seminal paper [44]. For $k \in \mathbb{Z}^{d}$, choose $\eta, \xi \in \mathbb{R}^{d}$ such that $|\xi|=|\eta|=1$ and $\xi \cdot \eta=\xi \cdot k=\eta \cdot k=0$. For $t \in \mathbb{R}$ define

$$
\begin{aligned}
& \zeta_{1}^{k, t}=-i(\pi k+t \xi)+\sqrt{t^{2}+\pi^{2}|k|^{2}} \eta, \\
& \zeta_{2}^{k, t}=-i(\pi k-t \xi)-\sqrt{t^{2}+\pi^{2}|k|^{2}} \eta
\end{aligned}
$$

so that

$$
\zeta_{j}^{k, t} \cdot \zeta_{j}^{k, t}=0 \quad \text { for } j=1,2, \quad \zeta_{1}^{k, t}+\zeta_{2}^{k, t}=-2 \pi i k .
$$

For every $t \geqslant c$ we can construct a solution $\psi^{k, t}$ of (1) in $\mathbb{R}^{d}$ (with $q$ extended to $\mathbb{R}^{d}$ by zero) of the form

$$
\psi^{k, t}(x)=\psi\left(x, \zeta_{1}^{k, t}\right)=e^{\zeta_{1}^{k, t} \cdot x}\left(1+r^{k, t}(x)\right), \quad x \in \mathbb{R}^{d},
$$


in which the error term $r^{k, t}$ satisfies the estimates

$$
\begin{gathered}
\left\|r^{k, t}\right\|_{L^{2}\left(\mathbb{T}^{d}\right)} \leqslant \frac{c}{t}, \\
\left\|r^{k, t}\right\|_{L^{p}\left(\mathbb{T}^{d}\right)} \leqslant c, \\
\left\|\nabla r^{k, t}\right\|_{L^{2}\left(\mathbb{T}^{d}\right)} \leqslant c .
\end{gathered}
$$

The first two bounds are given in [40] (see Theorem 4.1 and Lemma 5.5). The third estimate is not explicitly mentioned in this paper, but follows immediately from (5) and (7) and using standard energy estimates for elliptic equations. (The error term $r^{k, t}$ solves $-\Delta r^{k, t}=\operatorname{div}\left(\zeta_{1}^{k, t} r^{k, t}\right)+q\left(r^{k, t}-1\right)$ in a ball $B \supseteq \mathbb{T}^{d}$, and so

$$
\left\|\nabla r^{k, t}\right\|_{L^{2}\left(\mathbb{T}^{d}\right)} \leqslant c\left(\left|\zeta_{1}^{k, t}\right|\left\|r^{k, t}\right\|_{L^{2}(B)}+R\left\|r^{k, t}-1\right\|_{L^{2}(B)}+\left\|r^{k, t}\right\|_{L^{2}(B)}\right) \leqslant c
$$

where we used estimate ( 7$)$ in $B$.) In the three-dimensional case, estimate (8) was proven also with $p=+\infty$ [43]; the use of this version would slightly simplify some of the derivations below.

The solutions $\psi^{k, t}$ are known as exponentially growing solutions, Faddeev-type solutions [23] or CGO solutions. Note that $e^{\zeta_{2}^{k, t_{k}} \cdot x}$ is harmonic in $\mathbb{R}^{d}$.

It is useful to consider an ordering of the frequencies in $\mathbb{Z}^{d}$, namely a bijective $\operatorname{map} \rho: \mathbb{N} \rightarrow \mathbb{Z}^{d}, l \mapsto k_{l}$. For each $k \in \mathbb{Z}^{d}$ fix $t_{k} \geqslant c$ and define the measurement operator $U: L^{\infty}\left(\mathbb{T}^{d}\right) \rightarrow \ell^{\infty}$ by

$$
\begin{aligned}
(U(q))_{l} & :=\int_{\mathbb{T}^{d}} q(x) e^{\zeta_{2}^{k_{l}, t_{k_{l}}} \cdot x} \psi^{k_{l}, t_{k_{l}}}(x) d x \\
& =\left\langle e^{\zeta_{2} t^{k_{k_{l}}} \cdot x},\left(\Lambda_{q}-\Lambda_{0}\right) \psi^{k_{l}, t_{k_{l}}}\right\rangle_{H^{1 / 2}(\partial \Omega) \times H^{-1 / 2}(\partial \Omega)},
\end{aligned}
$$

where the second identity follows from (4) (and is valid only whenever (2) holds true and $q=0$ almost everywhere in $\mathbb{T}^{d} \backslash \Omega$ ). Note that the operator $U$ is nonlinear, since the solution $\psi^{k_{l}, t_{k_{l}}}$ depends on $q$. In the literature, this operator is also known as nonlinear Fourier transform or generalized scattering transform or amplitude. Using the same ordering of $\mathbb{Z}^{d}$, we define the discrete Fourier transform $F: L^{2}\left(\mathbb{T}^{d}\right) \rightarrow \ell^{2}$ by

$$
F(q):=\int_{\mathbb{T}^{d}} q(x) e^{-2 \pi i k_{l} \cdot x} d x=\left(\left\langle q, e_{k_{l}}\right\rangle\right)_{l},
$$

where $e_{k}(x)=e^{2 \pi i k \cdot x}$, and the nonlinear operator $B: L^{\infty}\left(\mathbb{T}^{d}\right) \rightarrow \ell^{\infty}$ by

$$
B(q):=\left(\left\langle q, e_{k_{l}} r_{l}\right\rangle\right)_{l}
$$

where $r_{l}=r^{k_{l}, t_{k_{l}}}$, so that $U=F+B$. 
The first global uniqueness proof for the 3D Calderón problem [44] consisted in showing that $U \rightarrow F$ as the parameter $t \rightarrow+\infty$. In our case we cannot do that, since we want to be able to select a finite number of measurements from the operator $U$, which depends on the choice of the fixed parameters $t_{k_{l}}$.

In order to prove a uniqueness result with a finite number of measurements we will use a fixed-point argument, based on the following lemma.

LEMma 1. Take $s>d p / 2(p-d)$. There exists $c^{\prime}>0$ depending only on $d, s, p$ and $R$, such that if $t_{k}=c^{\prime}\left(|k|^{s}+1\right)$ for every $k \in \mathbb{Z}^{d}$ then

$$
\left\|B\left(q_{2}\right)-B\left(q_{1}\right)\right\|_{\ell^{2}} \leqslant \frac{1}{2}\left\|q_{2}-q_{1}\right\|_{L^{2}\left(\mathbb{T}^{d}\right)}
$$

for every $q_{1}, q_{2} \in L^{\infty}\left(\mathbb{T}^{d}\right)$ such that $\left\|q_{i}\right\|_{L^{\infty}\left(\mathbb{T}^{d}\right)} \leqslant R, i=1$, 2 . In other words, the operator $B$ restricted to the closed ball $\bar{B}(0, R)$ of $L^{\infty}\left(\mathbb{T}^{d}\right)$ is a contraction.

Proof. We have, from the definition of $B$,

$$
\left(B\left(q_{2}\right)-B\left(q_{1}\right)\right)_{l}=\left\langle q_{2}-q_{1}, e_{l} r_{l}\left(q_{2}\right)\right\rangle+\left\langle q_{1}, e_{l}\left(r_{l}\left(q_{2}\right)-r_{l}\left(q_{1}\right)\right)\right\rangle,
$$

where we called $e_{l}=e_{k_{l}}$ and emphasized the dependence on $q_{i}$. By (7) and the assumption $t_{k}=c^{\prime}\left(|k|^{s}+1\right)$, we obtain

$$
\begin{aligned}
& \left|\left(B\left(q_{2}\right)-B\left(q_{1}\right)\right)_{l}\right| \leqslant\left\|q_{2}-q_{1}\right\|_{L^{2}\left(\mathbb{T}^{d}\right)}\left\|r_{l}\left(q_{2}\right)\right\|_{L^{2}\left(\mathbb{T}^{d}\right)} \\
& \quad+\left\|q_{1}\right\|_{L^{2}\left(\mathbb{T}^{d}\right)}\left\|r_{l}\left(q_{2}\right)-r_{l}\left(q_{1}\right)\right\|_{L^{2}\left(\mathbb{T}^{d}\right)} \\
& \quad \leqslant\left\|q_{2}-q_{1}\right\|_{L^{2}\left(\mathbb{T}^{d}\right)} \frac{c}{c^{\prime}\left(\left|k_{l}\right|^{s}+1\right)}+R\left\|r_{l}\left(q_{2}\right)-r_{l}\left(q_{1}\right)\right\|_{L^{2}\left(\mathbb{T}^{d}\right)} .
\end{aligned}
$$

Now, following [44], let $L w=\Delta w+\zeta_{1}^{k_{l} t_{k_{l}}} \cdot \nabla w$. The remainders $r_{l}\left(q_{i}\right)$ satisfy the equations

$$
\operatorname{Lr}_{l}\left(q_{i}\right)-q_{i} r_{l}\left(q_{i}\right)=q_{i}, \quad i=1,2,
$$

so that the difference $r_{l}=r_{l}\left(q_{2}\right)-r_{l}\left(q_{1}\right)$ satisfies

$$
L r_{l}-q_{2} r_{l}=\left(q_{2}-q_{1}\right)\left(1+r_{l}\left(q_{1}\right)\right) \text { in } \mathbb{R}^{d} .
$$

Applying [40, Theorem 4.1] to (13) gives

$$
\begin{aligned}
\left\|r_{l}\left(q_{2}\right)-r_{l}\left(q_{1}\right)\right\|_{L^{2}\left(\mathbb{T}^{d}\right)} & \leqslant c \frac{\left\|\left(q_{2}-q_{1}\right)\left(1+r_{l}\left(q_{1}\right)\right)\right\|_{L^{2 p /(p+2)}\left(\mathbb{T}^{d}\right)}}{\left|t_{k_{l}}\right|^{1-(d / p)}} \\
& \leqslant c \frac{\left\|\left(q_{2}-q_{1}\right)\right\|_{L^{2}\left(\mathbb{T}^{d}\right)}\left\|1+r_{l}\left(q_{1}\right)\right\|_{L^{p}\left(\mathbb{T}^{d}\right)}}{\left|t_{k_{l}}\right|^{1-(d / p)}} .
\end{aligned}
$$


As a consequence, by (8) we obtain

$$
\left\|r_{l}\left(q_{2}\right)-r_{l}\left(q_{1}\right)\right\|_{L^{2}\left(\mathbb{T}^{d}\right)} \leqslant \frac{c}{\left(c^{\prime}\left(\left|k_{l}\right|^{s}+1\right)\right)^{1-(d / p)}}\left\|q_{2}-q_{1}\right\|_{L^{2}\left(\mathbb{T}^{d}\right)} .
$$

Inserting this estimate into (12) yields

$$
\left|\left(B\left(q_{2}\right)-B\left(q_{1}\right)\right)_{l}\right| \leqslant \frac{c}{\left(c^{\prime}\left(\left|k_{l}\right|^{s}+1\right)\right)^{1-(d / p)}}\left\|q_{2}-q_{1}\right\|_{L^{2}\left(\mathbb{T}^{d}\right)} .
$$

Since $2 s-2 s d / p>d$, the series

$$
\gamma_{s}^{2}:=\sum_{l \in \mathbb{N}} \frac{1}{\left(\left|k_{l}\right|^{s}+1\right)^{2-(2 d / p)}}=\sum_{k \in \mathbb{Z}^{d}} \frac{1}{\left(|k|^{s}+1\right)^{2-(2 d / p)}}
$$

is convergent, and so we finally obtain

$$
\left\|B\left(q_{2}\right)-B\left(q_{1}\right)\right\|_{\ell^{2}} \leqslant \frac{c \gamma_{s}}{\left(c^{\prime}\right)^{1-(d / p)}}\left\|q_{2}-q_{1}\right\|_{L^{2}\left(\mathbb{T}^{d}\right)} .
$$

Choosing $c^{\prime} \geqslant\left(2 c \gamma_{s}\right)^{p /(p-d)}$ yields the desired result.

The next result shows that, given a known finite dimensional subspace $\mathcal{W}$ of $L^{\infty}(\Omega)$, there exists a number $N$ such that two potentials in $\mathcal{W}$ satisfying $\left(U\left(q_{1}\right)\right)_{l}=\left(U\left(q_{2}\right)\right)_{l}$ for $l=1, \ldots, N$ must coincide. Let $P_{N}: \ell^{\infty} \rightarrow \ell^{\infty}$ be the projection onto the first $N$ components, namely $P_{N}\left(a_{1}, a_{2}, \ldots\right)=\left(a_{1}, \ldots\right.$, $\left.a_{N}, 0,0, \ldots\right)$, and $P_{\mathcal{W}}: L^{2}\left(\mathbb{T}^{d}\right) \rightarrow L^{2}\left(\mathbb{T}^{d}\right)$ be the orthogonal projection onto $i(\mathcal{W})$, where $i: L^{\infty}(\Omega) \rightarrow L^{2}\left(\mathbb{T}^{d}\right)$ is the extension operator by zero. We also set $P_{\mathcal{W}}^{\perp}=I-P_{\mathcal{W}}$.

Proposition 1. Fix $t_{k}=c^{\prime}\left(|k|^{s}+1\right)$ for every $k \in \mathbb{Z}^{d}$ as in Lemma 1. There exists $N \in \mathbb{N}$ depending only on $\mathcal{W}$ such that the following is true. For any $q_{1}$, $q_{2} \in L^{\infty}\left(\mathbb{T}^{d}\right)$ with $\left\|q_{i}\right\|_{L^{\infty}\left(\mathbb{T}^{d}\right)} \leqslant R$ and $\left\|P_{\mathcal{W}}^{\perp} q_{i}\right\|_{L^{2}\left(\mathbb{T}^{d}\right)} \leqslant \varepsilon$ for some $\varepsilon \geqslant 0$ we have

$$
\left\|q_{1}-q_{2}\right\|_{L^{2}\left(\mathbb{T}^{d}\right)} \leqslant 4\left\|P_{N} U\left(q_{1}\right)-P_{N} U\left(q_{2}\right)\right\|_{\ell^{2}}+8 \varepsilon
$$

Proof. From the identity $U=F+B$ and the assumptions on $q_{1}, q_{2}$, we readily obtain

$$
\begin{aligned}
P_{N} U\left(q_{1}\right)-P_{N} U\left(q_{2}\right) & =P_{N} F\left(q_{1}-q_{2}\right)+P_{N}\left(B\left(q_{1}\right)-B\left(q_{2}\right)\right) \\
& =F\left(q_{1}-q_{2}\right)-P_{N}^{\perp} F\left(q_{1}-q_{2}\right)+P_{N}\left(B\left(q_{1}\right)-B\left(q_{2}\right)\right),
\end{aligned}
$$

where $P_{N}^{\perp}=I-P_{N}$, and thus

$$
\begin{aligned}
F\left(q_{1}-q_{2}\right)= & \left(P_{N} U\left(q_{1}\right)-P_{N} U\left(q_{2}\right)\right) \\
& +P_{N}^{\perp} F\left(q_{1}-q_{2}\right)-P_{N}\left(B\left(q_{1}\right)-B\left(q_{2}\right)\right) .
\end{aligned}
$$

Using the fact that $F$ is unitary and Lemma 1 we have 


$$
\begin{aligned}
& \left\|q_{1}-q_{2}\right\|_{L^{2}\left(\mathbb{T}^{d}\right)} \\
& \quad=\left\|F\left(q_{1}-q_{2}\right)\right\|_{\ell^{2}} \\
& \quad \leqslant\left\|P_{N} U\left(q_{1}\right)-P_{N} U\left(q_{2}\right)\right\|_{\ell^{2}}+\left\|P_{N}^{\perp} F\left(q_{1}-q_{2}\right)\right\|_{\ell^{2}}+\left\|P_{N}\left(B\left(q_{2}\right)-B\left(q_{1}\right)\right)\right\|_{\ell^{2}} \\
& \quad \leqslant\left\|P_{N} U\left(q_{1}\right)-P_{N} U\left(q_{2}\right)\right\|_{\ell^{2}}+\left\|P_{N}^{\perp} F\left(q_{1}-q_{2}\right)\right\|_{\ell^{2}}+\frac{1}{2}\left\|q_{1}-q_{2}\right\|_{L^{2}\left(\mathbb{T}^{d}\right)},
\end{aligned}
$$

which gives, using the fact that $\left\|P_{\mathcal{W}}^{\perp} q_{i}\right\|_{L^{2}\left(\mathbb{T}^{d}\right)} \leqslant \varepsilon$,

$$
\left\|q_{1}-q_{2}\right\|_{L^{2}\left(\mathbb{T}^{d}\right)} \leqslant 2\left\|P_{N}^{\perp} F P_{\mathcal{W}}\left(q_{1}-q_{2}\right)\right\|_{\ell^{2}}+2\left\|P_{N} U\left(q_{1}\right)-P_{N} U\left(q_{2}\right)\right\|_{\ell^{2}}+4 \varepsilon .
$$

Now, since $P_{N}^{\perp} \rightarrow 0$ strongly as $N \rightarrow \infty$ and $F P_{\mathcal{W}}$ is a finite rank operator, there exists $N$ such that

$$
\left\|P_{N}^{\perp} F P_{\mathcal{W}}\right\|_{L^{2}\left(\mathbb{T}^{d}\right) \rightarrow \ell^{2}} \leqslant \frac{1}{4} .
$$

This immediately yields the final estimate

$$
\left\|q_{1}-q_{2}\right\|_{L^{2}\left(\mathbb{T}^{d}\right)} \leqslant 4\left\|P_{N} U\left(q_{1}\right)-P_{N} U\left(q_{2}\right)\right\|_{\ell^{2}}+8 \varepsilon .
$$

REMARK 4. It is natural to wonder whether one may extend the uniqueness result presented in this paper to the case when $\mathcal{W}$ is a finite dimensional submanifold of $L^{\infty}(\Omega)$. While this remains a very interesting open question to investigate, it is clear that, in such generality, the current proof would not work. Indeed, when $\Omega=\mathbb{T}^{d}$, for the one-dimensional manifold

$$
\mathcal{W}=\left\{x \mapsto e^{2 \pi i \xi x_{1}}: \xi \in \mathbb{R}\right\} \subseteq L^{\infty}\left(\mathbb{T}^{d}\right),
$$

we immediately have that

$$
\sup _{q \in \mathcal{W}}\left\|P_{N}^{\perp} F q\right\|_{\ell^{2}}=1,
$$

and so an inequality like (14) cannot hold.

The next result is a more precise version of Theorem 1, where we show how having the same boundary measurements yields $P_{N} U\left(q_{1}\right)=P_{N} U\left(q_{2}\right)$.

THEOREM 3. Take $d \geqslant 3$ and let $\Omega \subseteq \mathbb{R}^{d}$ be a bounded Lipschitz domain and $\mathcal{W} \subseteq L^{\infty}(\Omega)$ be a finite dimensional subspace. There exists $N \in \mathbb{N}$ such that the following is true.

Take $R>0$ and $q_{1}, q_{2} \in \mathcal{W}$ satisfying $\left\|q_{j}\right\|_{L^{\infty}(\Omega)} \leqslant R$ and $(2)(j=1,2)$ and let $f_{l}=\left.\psi_{1}^{k_{l}, t_{k_{l}}}\right|_{\partial \Omega}$, where $\psi_{1}^{k_{l}, t_{k_{l}}}, l \in \mathbb{N}$, are the CGO solutions corresponding to $q_{1}$.

$$
\text { If } \Lambda_{q_{1}} f_{l}=\Lambda_{q_{2}} f_{l} \text { for } l=1, \ldots, N \text {, then } q_{1}=q_{2} \text {. }
$$


Proof. Let $\psi_{j}^{l}=\psi_{j}^{k_{l}, t_{k_{l}}}$ be the CGO solutions corresponding to $q_{j}, j=1,2$ and $N$ be as in Proposition 1. We claim that $\left.\psi_{1}^{l}\right|_{\partial \Omega}=\left.\psi_{2}^{l}\right|_{\partial \Omega}$, for $l=1, \ldots, N$. Indeed, $\left.\psi_{j}^{l}\right|_{\partial \Omega}$ can be characterized as the unique solution in $H^{1 / 2}(\partial \Omega)$ of the boundary integral equation

$$
\psi_{j}^{l}(x)=e^{\zeta_{1}^{k_{1}, t_{l} l} \cdot x}+\int_{\partial \Omega} G\left(x-y, \zeta_{1}^{k_{l}, t_{k_{l}}}\right)\left(\Lambda_{q_{j}}-\Lambda_{0}\right) \psi_{j}^{l}(y) d \sigma(y), \quad x \in \partial \Omega,
$$

for $j=1,2$, where $G(x, \zeta)$ is the Faddeev-Green function; see [37, Proposition 2], [35, Theorem 1.4], and [36, Theorem 5]. Note that we only need [36] to extend the results of [35] to Lipschitz domains and to take $H^{1 / 2}(\partial \Omega)$ as domain for the boundary integral equation (15).

Thus, for $l=1, \ldots, N$, since $\Lambda_{q_{1}}\left(\psi_{1}^{l}\right)=\Lambda_{q_{2}}\left(\psi_{1}^{l}\right),\left.\psi_{1}^{l}\right|_{\partial \Omega}$ satisfies

$$
\psi_{1}^{l}(x)=e^{\zeta_{1}^{k_{1}, t_{k_{l}}} \cdot x}+\int_{\partial \Omega} G\left(x-y, \zeta_{1}^{k_{l}, t_{k_{l}}}\right)\left(\Lambda_{q_{2}}-\Lambda_{0}\right) \psi_{1}^{l}(y) d \sigma(y), \quad x \in \partial \Omega,
$$

which yields $\left.\psi_{1}^{l}\right|_{\partial \Omega}=\left.\psi_{2}^{l}\right|_{\partial \Omega}$ because of the unique solvability of (15) for $j=2$. This readily gives

$$
\begin{aligned}
\left(U\left(q_{1}\right)\right)_{l} & =\left\langle e^{\zeta_{2}^{k_{l}, t_{l}} \cdot x},\left(\Lambda_{q_{1}}-\Lambda_{0}\right) \psi_{1}^{l}\right\rangle_{H^{1 / 2}(\partial \Omega) \times H^{-1 / 2}(\partial \Omega)} \\
& =\left\langle e^{\zeta_{2}^{k_{l}, t_{l}} \cdot x},\left(\Lambda_{q_{2}}-\Lambda_{0}\right) \psi_{2}^{l}\right\rangle_{H^{1 / 2}(\partial \Omega) \times H^{-1 / 2}(\partial \Omega)} \\
& =\left(U\left(q_{2}\right)\right)_{l},
\end{aligned}
$$

for $l=1, \ldots, N$, that is $P_{N} U\left(q_{1}\right)=P_{N} U\left(q_{2}\right)$. Finally, by Proposition 1 with $\varepsilon=0$ we obtain $q_{1}=q_{2}$.

We now pass to the proof of the stability estimate.

Proof of Theorem 2. During the proof, several positive constants depending only on $\Omega, R, \alpha$ and $\rho$ will be denoted by the same letter $C$. As in Theorem 3, we let $f_{l}=\left.\psi_{1}^{k_{l}, t_{l}}\right|_{\partial \Omega}$, for $l=1, \ldots, N$, where we make the choices $s=d / 2+\alpha d$ and $p=d+1+d / 2 \alpha$, so that $s>d p / 2(p-d)$. We also choose a particular ordering $\rho: l \in \mathbb{N} \mapsto k_{l} \in \mathbb{Z}^{d}$ of the frequencies: suppose that

$$
\left|k_{l}\right| \leqslant C_{\rho} l^{1 / d} \text { for some } C_{\rho}>0 .
$$

From the definition of $U$, using [38, Theorem 1], for $l=1, \ldots, N$ we have the identity:

$$
\left(U\left(q_{1}\right)\right)_{l}-\left(U\left(q_{2}\right)\right)_{l}=\left\langle\psi_{2}\left(\cdot, \zeta_{2}^{k_{l}, t_{l}}\right),\left(\Lambda_{q_{1}}-\Lambda_{q_{2}}\right) \psi_{1}^{l}\right\rangle_{H^{1 / 2}(\partial \Omega) \times H^{-1 / 2}(\partial \Omega)} .
$$


This is a particular case of identity (2.8) of [38], with a different notation: $U(q)_{l}$ corresponds to $h\left(-i \zeta_{1}^{k_{l}, t_{k_{l}}}, i \zeta_{2}^{k_{l}, t_{k_{l}}}\right), \psi(x, \zeta)$ corresponds to $\psi(x,-i \zeta)$ and the operator $\Lambda_{q}$ to $\Phi$.

We then readily obtain

$$
\left|\left(U\left(q_{1}\right)\right)_{l}-\left(U\left(q_{2}\right)\right)_{l}\right| \leqslant\left\|\psi_{2}\left(\cdot, \zeta_{2}^{k_{l}, t_{k_{l}}}\right)\right\|_{H^{1 / 2}(\partial \Omega)}\left\|\left(\Lambda_{q_{1}}-\Lambda_{q_{2}}\right) \psi_{1}^{l}\right\|_{H^{-1 / 2}(\partial \Omega)} .
$$

The first term can be bounded using the trace theorem [22]:

$$
\begin{aligned}
& \left\|\psi_{2}\left(\cdot, \zeta_{2}^{k_{l}, t_{k_{l}}}\right)\right\|_{H^{1 / 2}(\partial \Omega)} \leqslant\left\|\psi_{2}\left(\cdot, \zeta_{2}^{k_{l}, t_{k_{l}}}\right)\right\|_{H^{1}(\Omega)} \\
& \leqslant\left\|e^{\zeta_{2}^{k_{l}, t_{k_{l}}} \cdot x}\left(1+r_{l}\left(q_{2}, \zeta_{2}^{k_{l}, t_{k_{l}}}\right)\right)\right\|_{H^{1}(\Omega)} \\
& \leqslant C e^{C\left|k_{l}\right|^{s}}\left(\|1\|_{H^{1}(\Omega)}+\left\|r_{l}\left(q_{2}, \zeta_{2}^{k_{l}, t_{k_{l}}}\right)\right\|_{H^{1}(\Omega)}\right) \\
& \leqslant C e^{C l^{s / d}} \text {, }
\end{aligned}
$$

where we used (6), (16), (7), the assumptions on $t_{k_{l}}$ and the boundedness of $\Omega$.

We have found

$$
\left|\left(U\left(q_{1}\right)\right)_{l}-\left(U\left(q_{2}\right)\right)_{l}\right| \leqslant C e^{C l^{s / d}}\left\|\left(\Lambda_{q_{1}}-\Lambda_{q_{2}}\right) \psi_{1}^{l}\right\|_{H^{-1 / 2}(\partial \Omega)},
$$

for $l=1, \ldots, N$, which gives

$$
\begin{aligned}
\left\|P_{N} U\left(q_{1}\right)-P_{N} U\left(q_{2}\right)\right\|_{\ell^{2}} & \leqslant C e^{C N^{s / d}} \sqrt{\sum_{l=1}^{N}\left\|\left(\Lambda_{q_{1}}-\Lambda_{q_{2}}\right) \psi_{1}^{l}\right\|_{H^{-1 / 2}(\partial \Omega)}^{2}} \\
& =C e^{C N^{1 / 2+\alpha}}\left\|\left(\left(\Lambda_{q_{1}}-\Lambda_{q_{2}}\right) \psi_{1}^{l}\right)_{l=1}^{N}\right\|_{H^{-1 / 2}(\partial \Omega)^{N}},
\end{aligned}
$$

where we set $\left\|\left(\varphi_{l}\right)_{l}\right\|_{H^{-1 / 2}(\partial \Omega)^{N}}^{2}:=\sum_{l=1}^{N}\left\|\varphi_{l}\right\|_{H^{-1 / 2}(\partial \Omega)}^{2}$. The proof follows from the last estimate and Proposition 1 with $\varepsilon=0$.

REMARK 5. The proof of Theorem 2 makes use of a particular ordering of the frequencies $\rho$ satisfying (16). This yields the explicit stability constant $e^{C N^{1 / 2+\alpha}}$. However, the stability result remains valid for an arbitrary ordering $\rho$, and the constant becomes

$$
\exp \left(C \max \left(\left|k_{1}\right|^{s}, \ldots,\left|k_{N}\right|^{s}\right)\right),
$$

where $s$ is an arbitrary parameter larger than $d / 2$.

REMARK 6. Theorem 2 remains true if the assumption $q_{1}, q_{2} \in \mathcal{W}$ is relaxed to $\left\|P_{\mathcal{W}}^{\perp} q_{i}\right\|_{L^{2}\left(\mathbb{T}^{d}\right)} \leqslant \varepsilon$ for some $\varepsilon>0$. It is enough to apply Proposition 1 with $\varepsilon>0$, and the stability estimate becomes

$$
\left\|q_{2}-q_{1}\right\|_{L^{2}(\Omega)} \leqslant e^{C N^{1 / 2+\alpha}}\left\|\left(\Lambda_{q_{2}} f_{j}-\Lambda_{q_{1}} f_{j}\right)_{j=1}^{N}\right\|_{H^{-1 / 2}(\partial \Omega)^{N}}+8 \varepsilon .
$$


In other words, the reconstruction error is controlled by the measurement error and by the error in the approximation of the unknown by an element of the subspace $\mathcal{W}$. This is relevant in practice: the unknown potential will in general only be well-approximated by an element of a finite dimensional space, but may not belong to it.

Proof of Corollary 1. Using the Liouville transformation $u=\tilde{u} / \sqrt{\sigma}$, if $u$ solves the conductivity equation $\operatorname{div}(\sigma \nabla u)=0$, then $\tilde{u}$ solves the Schrödinger equation $(-\Delta+q) \tilde{u}=0$, with $q=\Delta \sqrt{\sigma} / \sqrt{\sigma}$.

Now, since we have $\sigma_{j}=1$ in a neighborhood of $\partial \Omega$, we have that $\Lambda_{\sigma_{j}}=\Lambda_{q_{j}}, q_{j}=\Delta \sqrt{\sigma_{j}} / \sqrt{\sigma_{j}}$, for $j=1,2$, because of the well-known identity $\Lambda_{q}=\sigma^{-1 / 2}\left(\Lambda_{\sigma}+(1 / 2)(\partial \sigma / \partial v)\right) \sigma^{-1 / 2}$, which follows from the Liouville transformation. Theorem 1 immediately yields $q_{1}=q_{2}$, and since $\sigma_{j}$ are solutions to $\left(\Delta-q_{j}\right) \sqrt{\sigma_{j}}=0$ in $\Omega$ with $\left.\sigma_{j}\right|_{\partial \Omega}=1$, we have $\sigma_{1}=\sigma_{2}$.

Proof of Corollary 2. Arguing as in the proof of [6, Theorem 1] we have the following identity

$$
\operatorname{div}\left(\left(\sigma_{1} \sigma_{2}\right)^{1 / 2} \nabla \log \left(\sigma_{1} / \sigma_{2}\right)\right)=2\left(\sigma_{1} \sigma_{2}\right)^{1 / 2}\left(q_{1}-q_{2}\right) \text { in } \Omega .
$$

Now, using (3) we find

$$
\left\|\sigma_{1}-\sigma_{2}\right\|_{L^{2}(\Omega)} \leqslant C(\lambda, \Omega)\left\|\log \left(\sigma_{1} / \sigma_{2}\right)\right\|_{L^{2}(\Omega)} \leqslant C(\lambda, \Omega)\left\|q_{1}-q_{2}\right\|_{L^{2}(\Omega)} .
$$

Thus, the proof follows from the estimate of Theorem 2 and the fact that $\Lambda_{\sigma_{j}}=$ $\Lambda_{q_{j}}, j=1,2$.

\section{Reconstruction}

The results of Section 2 can be used to design an iterative nonlinear reconstruction algorithm and to show that it is globally convergent. For simplicity, we consider directly $\Omega=\mathbb{T}^{d}$, but the general case may be handled by using the extension operator as above.

Given a finite dimensional subspace $\mathcal{W} \subseteq L^{\infty}\left(\mathbb{T}^{d}\right)$ and $R>0$, define

$$
\mathcal{W}_{R}:=\left\{q \in \mathcal{W}:\|q\|_{L^{\infty}\left(\mathbb{T}^{d}\right)} \leqslant R\right\},
$$

equipped with the $L^{2}$ norm. For $N \in \mathbb{N}$ and $y \in \ell^{2}$, we define the nonlinear operator $A: \mathcal{W}_{R} \rightarrow \mathcal{W}_{R}$ by

$$
A(q)=P_{\mathcal{W}_{R}}\left(F^{-1} y+F^{-1} P_{N}^{\perp} F q-F^{-1} P_{N} B(q)\right),
$$


where $F, B, P_{N}$ and $P_{N}^{\perp}$ were defined in Section 2 and $P_{\mathcal{W}_{R}}$ is the projection from $L^{2}\left(\mathbb{T}^{d}\right)$ onto the closed and convex set $\mathcal{W}_{R}$.

Now let $q \in \mathcal{W}_{R}$ satisfying (2) be the unknown potential and choose the number of measurements $N \in \mathbb{N}$ as in (14), that is so that

$$
\left\|P_{N}^{\perp} F P_{\mathcal{W}}\right\|_{L^{2}\left(\mathbb{T}^{d}\right) \rightarrow \ell^{2}} \leqslant \frac{1}{4} .
$$

Let $\left(f_{l}, \Lambda_{q}\left(f_{l}\right)\right)_{l=1}^{N}$ be the given boundary data, where $f_{l}=\psi^{k_{l}, t_{k_{l}}}$ are the CGO constructed in Section 2, and set $y=P_{N} U(q)$, which is directly computed from $\left(f_{l}, \Lambda_{q}\left(f_{l}\right)\right)_{l=1}^{N}$ thanks to (10).

The following nonlinear iterative reconstruction algorithm allows for the recovery of $q$ starting from the data $y$.

THEOREM 4. Under the above assumptions, let $q_{0} \in \mathcal{W}_{R}$ be any initial guess potential and define the sequence

$$
q_{n}=A\left(q_{n-1}\right), \quad n \geqslant 1 .
$$

Then we have the following convergence result:

$$
\left\|q-q_{n}\right\|_{L^{2}\left(\mathbb{T}^{d}\right)} \leqslant 4\left(\frac{3}{4}\right)^{n}\left\|q_{1}-q_{0}\right\|_{L^{2}\left(\mathbb{T}^{d}\right)}, \quad n \geqslant 1 .
$$

Proof. We claim that $A(q)=q$ and that $A$ is a contraction. Indeed, by using the identities $y=P_{N} U(q)$ and $U=F+B$ we readily derive

$$
\begin{aligned}
A(q) & =P_{\mathcal{W}_{R}}\left(F^{-1} P_{N} U(q)-F^{-1} P_{N} B(q)+F^{-1} P_{N}^{\perp} F q\right) \\
& =P_{\mathcal{W}_{R}}\left(F^{-1} P_{N} F q+F^{-1} P_{N}^{\perp} F q\right) \\
& =q .
\end{aligned}
$$

Further, it is a straightforward consequence of the fact that $P_{\mathcal{W}_{R}}$ is Lipschitz (with constant 1, by the Hilbert projection theorem), of Lemma 1 and of assumption (18) that the operator $A$ is a contraction on $\mathcal{W}_{R}$, namely

$$
\left\|A\left(q_{2}\right)-A\left(q_{1}\right)\right\|_{L^{2}\left(\mathbb{T}^{d}\right)} \leqslant \frac{3}{4}\left\|q_{2}-q_{1}\right\|_{L^{2}\left(\mathbb{T}^{d}\right)}, \quad q_{1}, q_{2} \in \mathcal{W}_{R}
$$

The result is now an immediate consequence of the Banach fixed-point theorem, since $\mathcal{W}_{R}$ is a complete metric space with the distance given by the $L^{2}$ norm.

Some comments on this result are in order.

- The exponential rate guarantees a very fast convergence of the iterates to the unknown $q$, and is consistent with the Lipschitz stability of the inverse problem given in Theorem 2. 
- We have not presented the details of the corresponding reconstruction algorithm for the Calderón problem, which can be easily obtained by using the Liouville transformation $q=\Delta \sqrt{\sigma} / \sqrt{\sigma}, v=u / \sqrt{\sigma}$ as in the proof of Corollaries 1 and 2, in order to formulate Calderón problem as an inverse boundary value problem for the Schödinger equation (1).

\section{Examples of subspaces $\mathcal{W}$}

As mentioned in Remark 1, the number $N$ of required measurements to have global uniqueness and stability depends only on the subspace $\mathcal{W} \subseteq L^{\infty}(\Omega)$ of the potentials. The dependence is explicit via condition equation (14):

$$
\left\|P_{N}^{\perp} F P_{\mathcal{W}}\right\|_{L^{2}\left(\mathbb{T}^{d}\right) \rightarrow \ell^{2}} \leqslant \frac{1}{4},
$$

in which $\mathcal{W}$, with an abuse of notation, denotes $i(\mathcal{W})$, where $i: L^{\infty}(\Omega) \rightarrow$ $L^{2}\left(\mathbb{T}^{d}\right)$ is the extension operator by zero. This condition appears in the literature on signal reconstruction from low frequency Fourier measurements [2, 41]: it is strictly related with the balancing property, a fundamental concept in sampling theory and CS in infinite dimension $[1,3,4]$.

It is worth considering some relevant examples of subspaces $\mathcal{W}$ and to compute the corresponding $N$ as a function of $\operatorname{dim} \mathcal{W}$. In other words, given $\mathcal{W}$, how many measurements $N$ should we take to have global uniqueness and stability for the inverse problem?

4.1. Bandlimited potentials. The simplest situation one may consider is with bandlimited potentials $q$ in $L^{\infty}\left(\mathbb{T}^{d}\right)$ (for simplicity, we set $\Omega=\mathbb{T}^{d}$ ). More precisely, the subspace $\mathcal{W}$ is given by

$$
\mathcal{W}=\left\{q \in L^{\infty}\left(\mathbb{T}^{d}\right): \hat{q}(k)=0 \text { for every } k \in \mathbb{Z}^{d},\|k\|_{\infty}>B\right\},
$$

where $\hat{q}(k):=\left\langle q, e_{k}\right\rangle$ is the Fourier transform and $B \in \mathbb{N}$. In other words, we have

$$
\mathcal{W}=\operatorname{span}\left\{e_{k}: k \in \mathbb{Z}^{d},\|k\|_{\infty} \leqslant B\right\} \subseteq L^{\infty}\left(\mathbb{T}^{d}\right),
$$

so that $\operatorname{dim} \mathcal{W}=(2 B+1)^{d}$.

It is convenient to choose the ordering $\rho: \mathbb{N} \rightarrow \mathbb{Z}^{d}$ in such a way that the frequencies in $\left\{k \in \mathbb{Z}^{d}:\|k\|_{\infty} \leqslant B\right\}$ come first, namely

$$
\rho(\{1, \ldots, \operatorname{dim} \mathcal{W}\})=\left\{k \in \mathbb{Z}^{d}:\|k\|_{\infty} \leqslant B\right\} .
$$

Hence, by (11) we immediately have $(F q)_{l}=0$ for every $q \in \mathcal{W}$ and $l>\operatorname{dim} \mathcal{W}$. As a result, choosing $N=\operatorname{dim} \mathcal{W}$ gives $\left\|P_{N}^{\perp} F P_{\mathcal{W}}\right\|_{L^{2}\left(\mathbb{T}^{d}\right) \rightarrow \ell^{2}}=0$, and so (19) is automatically satisfied. This is the optimal situation: the number of required measurements equals the dimension of the subspace of the unknowns. 
4.2. Piecewise constant potentials. A relevant case for the applications is of piecewise constant potentials $[13]$ (see $[8,9,42]$ and references therein for related results).

We consider here a particular situation. Let $R_{1}, \ldots, R_{M} \subseteq \Omega$ be $M$ subdomains such that:

- each subdomain $R_{i}$ is a $d$-dimensional interval with side lengths

$$
a_{1}^{i} M^{-1 / d}, \ldots, a_{d}^{i} M^{-1 / d}
$$

where the scaling $M^{-1 / d}$ is put since the size of $\Omega$ is of order 1;

- the weights $a_{j}^{i}$ are such that $A \leqslant a_{j}^{i}$ for some $A \in\left(0, M^{1 / d} / \pi\right]$ and allow for different shapes of the subdomains;

- and the interiors of these subdomains are disjoint, namely $\stackrel{\circ}{R}_{i_{1}} \cap \stackrel{\circ}{R}_{i_{2}}=\emptyset$ for every $i_{1} \neq i_{2}$.

The subspace $\mathcal{W}$ is given by

$$
\mathcal{W}=\operatorname{span}\left\{\chi_{R_{1}}, \ldots, \chi_{R_{M}}\right\},
$$

where $\chi_{R}$ is the characteristic function of $R$.

The other important ingredient of (19) is the ordering of the frequencies $\rho: \mathbb{N} \rightarrow \mathbb{Z}^{d}, l \mapsto k_{l}$. Here we suppose that $\rho$ corresponds to the hyperbolic cross in $\mathbb{Z}^{d}$ [30, Example 5.12], namely

$$
l_{1} \leqslant l_{2} \quad \Longrightarrow \quad \prod_{j=1}^{d} \max \left(\left|\rho\left(l_{1}\right)_{j}\right|, 1\right) \leqslant \prod_{j=1}^{d} \max \left(\left|\rho\left(l_{2}\right)_{j}\right|, 1\right) \text {. }
$$

Recall that the Fourier transform $F: L^{2}\left(\mathbb{T}^{d}\right) \rightarrow \ell^{2}$ is defined by (11), where the frequencies are ordered according to $\rho$.

We now prove that the number of measurements needed to satisfy (19) (and so to have global uniqueness for the inverse problem considered) is proportional to $M^{4}$, up to $\log$ factors. It is worth observing that this is only a sufficient condition, and may not be necessary. Indeed, the use of the Cauchy-Schwarz inequality in equation (23) yields an additional $M$ factor, which could perhaps be removed arguing as in [41, Lemma 5.1], at least for a uniform partition of $\mathbb{T}^{d}$ made of $d$ cubes. The search for the optimal exponent goes beyond the scopes of this work, and is an interesting direction for future research.

PROPOSITION 2. Under the above assumptions, we have

$$
\left\|P_{N}^{\perp} F P_{\mathcal{W}}\right\|_{L^{2}\left(\mathbb{T}^{d}\right) \rightarrow \ell^{2}} \leqslant C \frac{\log ^{d-1}(N)}{\sqrt{N}} M^{2},
$$


for some $C>0$ depending only on $d, \rho$ and A. In particular, (19) is satisfied provided that

$$
\frac{N}{\log ^{2 d-2}(N)} \geqslant 16 C^{2} M^{4}
$$

Proof. Setting $\operatorname{sinc}(x)=\sin (x) / x$, a direct calculation shows that

$$
\left|F \chi_{R_{i}}(l)\right|=\prod_{j=1}^{d}\left|\operatorname{sinc}\left(\pi M^{-1 / d} a_{j}^{i} \rho(l)_{j}\right)\right| \leqslant \prod_{j=1}^{d} \min \left(\frac{1}{\left|\pi M^{-1 / d} a_{j}^{i} \rho(l)_{j}\right|}, 1\right) .
$$

Thus, we readily obtain

$$
\left|F \chi_{R_{i}}(l)\right| \leqslant \prod_{j=1}^{d} \frac{1}{\max \left(\left|\pi M^{-1 / d} A \rho(l)_{j}\right|, 1\right)}=\prod_{j=1}^{d} \frac{(A \pi)^{-1} M^{1 / d}}{\max \left(\left|\rho(l)_{j}\right|,(A \pi)^{-1} M^{1 / d}\right)} .
$$

Hence, in view of (20), we can apply [30, Lemma 5.13] and obtain

$$
\left|F \chi_{R_{i}}(l)\right| \leqslant(A \pi)^{-d} M \frac{1}{\prod_{j=1}^{d} \max \left(\left|\rho(l)_{j}\right|, 1\right)} \leqslant C(A, d, \rho) M \frac{\log ^{d-1}(l+1)}{l} .
$$

Take now $f \in \mathcal{W}$ with $\|f\|_{L^{2}\left(\mathbb{T}^{d}\right)}=1$. Since

$$
\left\{f_{i}=\frac{\sqrt{M}}{\sqrt{a_{1}^{i} \cdots a_{d}^{i}}} \chi_{R_{i}}: i=1, \ldots, M\right\}
$$

is an orthonormal basis of $\mathcal{W}$, we can write $f=\sum_{i=1}^{M} c_{i} f_{i}$ with $\sum_{i} c_{i}^{2}=1$. Thus we have

$$
|F f(l)| \leqslant \sum_{i=1}^{M}\left|c_{i}\right| \frac{\sqrt{M}}{\sqrt{a_{1}^{i} \cdots a_{d}^{i}}}\left|F \chi_{R_{i}}(l)\right| \leqslant \sqrt{M / A^{d}}\left(\sum_{i=1}^{M}\left|F \chi_{R_{i}}(l)\right|^{2}\right)^{1 / 2} .
$$

Therefore, (22) immediately yields $|F f(l)| \leqslant C(A, d, \rho) M^{2}\left(\log ^{d-1}(l+1) / l\right)$, and so

$$
\begin{aligned}
\left\|P_{N}^{\perp} F f\right\|_{\ell^{2}}^{2} & =\sum_{l=N+1}^{+\infty}|F f(l)|^{2} \\
& \leqslant C(A, d, \rho) M^{4} \sum_{l=N+1}^{+\infty} \frac{\log ^{2 d-2}(l+1)}{l^{2}} \\
& \leqslant C(A, d, \rho) M^{4} \frac{\log ^{2 d-2}(N)}{N} .
\end{aligned}
$$

Finally, this bound immediately implies (21). 
4.3. Potentials belonging to low-scale wavelet subspaces. Given the importance of wavelets in imaging, it is interesting to look at the case when $\mathcal{W}$ is a subspace of dimension $M$ given by wavelets below a certain scale. Under certain assumptions on the mother wavelet and the scaling function, one has $N=O(M)$ if $d=1$ [41, Lemma 5.1]. This result is expected to hold also in higher dimension, at least in the case of separable wavelets, for which the 1D proof should be easily generalizable (see also [30]). Therefore, if the unknown potential belongs to the space generated by the first $M$ wavelets (ordered according to the scale), $O(M)$ measurements are needed for the reconstruction (up to log factors), and so this estimate is substantially the best possible. It is worth observing that much fewer measurements are needed in this case than in the piecewise constant case.

\section{Conflict of interest declaration}

None.

\section{References}

[1] B. Adcock and A. C. Hansen, 'Generalized sampling and infinite-dimensional compressed sensing', Found. Comput. Math. 16 (2016), 1263-1323.

[2] B. Adcock, A. C. Hansen and C. Poon, 'On optimal wavelet reconstructions from Fourier samples: linearity and universality of the stable sampling rate', Appl. Comput. Harmon. Anal. 36 (2014), 387-415.

[3] B. Adcock, A. C. Hansen, C. Poon and B. Roman, 'Breaking the coherence barrier: a new theory for compressed sensing', Forum Math. Sigma 5 (2017), e4, 84.

[4] G. S. Alberti and M. Santacesaria, 'Infinite dimensional compressed sensing from anisotropic measurements and applications to inverse problems in PDE', Appl. Comput. Harmon. Anal. (2019), doi:10.1016/j.acha.2019.08.002.

[5] G. S. Alberti and M. Santacesaria, 'Infinite-dimensional inverse problems with finite measurements'. Preprint, 2019, arXiv:1906.10028.

[6] G. Alessandrini, 'Stable determination of conductivity by boundary measurements', Appl. Anal. 27 (1988), 153-172.

[7] G. Alessandrini, M. V. De Hoop and R. Gaburro, 'Uniqueness for the electrostatic inverse boundary value problem with piecewise constant anisotropic conductivities', Inverse Problems 33125013 (2017).

[8] G. Alessandrini, M. V. de Hoop, R. Gaburro and E. Sincich, 'Lipschitz stability for the electrostatic inverse boundary value problem with piecewise linear conductivities', J. Math. Pures Appl. 107(9) (2017), 638-664.

[9] G. Alessandrini and S. Vessella, 'Lipschitz stability for the inverse conductivity problem', Adv. Appl. Math. 35 (2005), 207-241.

[10] K. Astala and L. Päivärinta, 'Calderón's inverse conductivity problem in the plane', Ann. of Math. (2) 163(1) (2006), 265-299. 
[11] E. Beretta, M. V. de Hoop, E. Francini and S. Vessella, 'Stable determination of polyhedral interfaces from boundary data for the Helmholtz equation', Comm. Partial Differential Equations 40 (2015), 1365-1392.

[12] E. Beretta, M. V. de Hoop, E. Francini, S. Vessella and J. Zhai, 'Uniqueness and Lipschitz stability of an inverse boundary value problem for time-harmonic elastic waves', Inverse Problems 33 (2017), 035013, 27.

[13] E. Beretta, M. V. de Hoop and L. Qiu, 'Lipschitz stability of an inverse boundary value problem for a Schrödinger-type equation', SIAM J. Math. Anal. 45 (2013), 679-699.

[14] E. Beretta and E. Francini, 'Lipschitz stability for the electrical impedance tomography problem: the complex case', Comm. Partial Differential Equations 36 (2011), 1723-1749.

[15] E. Blåsten, O. Y. Imanuvilov and M. Yamamoto, 'Stability and uniqueness for a twodimensional inverse boundary value problem for less regular potentials', Inverse Probl. Imaging 9 (2015), 709-723.

[16] E. Blåsten and H. Liu, 'Recovering piecewise constant refractive indices by a single far-field pattern'. Preprint, 2017, arXiv:1705.00815.

[17] A. L. Bukhgeim, 'Recovering a potential from Cauchy data in the two-dimensional case', J. Inverse Ill-Posed Probl. 16 (2008), 19-33.

[18] A. P. Calderón, 'On an inverse boundary value problem', in Seminar on Numerical Analysis and its Applications to Continuum Physics (Rio de Janeiro, 1980) (Soc. Brasil. Mat., Rio de Janeiro, 1980), 65-73.

[19] P. Caro and K. M. Rogers, 'Global uniqueness for the calderón problem with lipschitz conductivities', Forum of Mathematics, Pi 4 (2016), E2. doi:10.1017/fmp.2015.9.

[20] A. Clop, D. Faraco and A. Ruiz, 'Stability of Calderón's inverse conductivity problem in the plane for discontinuous conductivities', Inverse Probl. Imaging 4 (2010), 49-91.

[21] M. Di Cristo and L. Rondi, 'Examples of exponential instability for inverse inclusion and scattering problems', Inverse Problems 19 (2003), 685.

[22] Z. Ding, 'A proof of the trace theorem of Sobolev spaces on Lipschitz domains', Proc. Amer. Math. Soc. 124 (1996), 591-600.

[23] L. D. Faddeev, 'Increasing solutions of the Schrödinger equation', Sov. Phys. Dok. 10 (1966), 1033-1035.

[24] A. Friedman and V. Isakov, 'On the uniqueness in the inverse conductivity problem with one measurement', Indiana Univ. Math. J. 38 (1989), 563-579.

[25] R. Gaburro and E. Sincich, 'Lipschitz stability for the inverse conductivity problem for a conformal class of anisotropic conductivities', Inverse Problems 31 (2015), 015008, 26.

[26] B. Haberman, 'Uniqueness in calderón's problem for conductivities with unbounded gradient', Commun. Math. Phys. 340 (2015), 639-659.

[27] B. Harrach, 'Uniqueness and Lipschitz stability in electrical impedance tomography with finitely many electrodes', Inverse Problems 35 (2019), 024005, 19.

[28] M. I. Isaev, 'Exponential instability in the Gel'fand inverse problem on the energy intervals', J. Inverse Ill-Posed Probl. 19 (2011), 453-472.

[29] M. Isaev and R. Novikov, 'Reconstruction of a potential from the impedance boundary map', Eur. J. Math. Comput. Appl. 1 (2013), 5-28.

[30] A. D. Jones, B. Adcock and A. C. Hansen, 'Analyzing the structure of multidimensional compressed sensing problems through coherence', Preprint, 2016, arXiv:1610.07497.

[31] E. L. Lakshtanov, R. G. Novikov and B. R. Vainberg, 'A global Riemann-Hilbert problem for two-dimensional inverse scattering at fixed energy', Rend. Istit. Mat. Univ. Trieste 48 (2016), $21-47$. 
[32] E. L. Lakshtanov, J. Tejero and B. R. Vainberg, 'Uniqueness in the inverse conductivity problem for complex-valued Lipschitz conductivities in the plane', SIAM J. Math. Anal. 49 (2017), 3766-3775.

[33] N. Mandache, 'Exponential instability in an inverse problem for the Schrödinger equation', Inverse Problems 17 (2001), 1435.

[34] J. L. Mueller and S. Siltanen, Linear and Nonlinear Inverse Problems with Practical Applications, Computational Science \& Engineering, 10 (Society for Industrial and Applied Mathematics (SIAM), Philadelphia, PA, 2012).

[35] A. I. Nachman, 'Reconstructions from boundary measurements', Ann. of Math. (2) 128 (1988), 531-576.

[36] A. I. Nachman, 'Global uniqueness for a two-dimensional inverse boundary value problem', Ann. of Math. (2) 143 (1996), 71-96.

[37] R. G. Novikov, 'A multidimensional inverse spectral problem for the equation $-\Delta \psi+(v(x)-$ $E u(x)) \psi=0$ ', Funct. Anal. Appl. 22 (1988), 263-272.

[38] R. G. Novikov, 'Formulae and equations for finding scattering data from the Dirichlet-toNeumann map with nonzero background potential', Inverse Problems 21 (2004), 257.

[39] R. G. Novikov and M. Santacesaria, 'A global stability estimate for the Gel'fand-Calderón inverse problem in two dimensions', J. Inverse Ill-Posed Probl. 18 (2010), 765-785.

[40] L. Päivärinta, 'Analytic methods for inverse scattering theory', in New Analytic and Geometric Methods in Inverse Problems (Springer, Berlin, 2004), 165-185.

[41] C. Poon, 'A consistent and stable approach to generalized sampling', J. Fourier Anal. Appl. 20 (2014), 985-1019.

[42] L. Rondi, 'A remark on a paper by G. Alessandrini and S. Vessella: "Lipschitz stability for the inverse conductivity problem", Adv. Appl. Math. 35(2) (2005), 207-241. mr2152888, Adv. in Appl. Math., 36 (2006), 67-69.

[43] V. Serov, 'Inverse fixed energy scattering problem for the generalized nonlinear Schrödinger operator', Inverse Problems 28 (2012), 025002, 11.

[44] J. Sylvester and G. Uhlmann, 'A global uniqueness theorem for an inverse boundary value problem', Ann. of Math. (2) 125 (1987), 153-169. 